\title{
Implementation of the Windowed Multipole Method in Shift
}

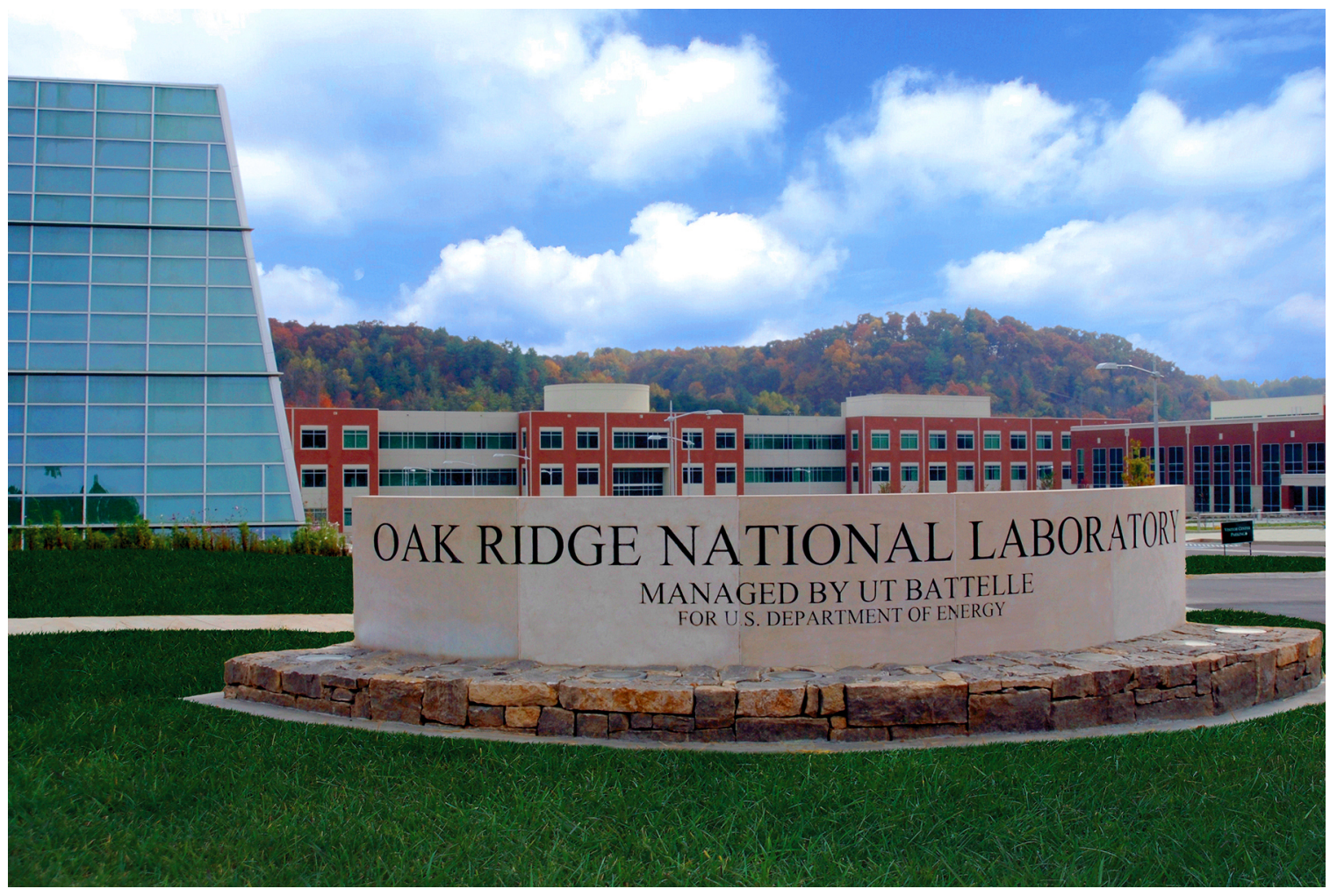

Elliott D. Biondo

Approved for public release.

Distribution is unlimited.

July 15, 2021 


\section{DOCUMENT AVAILABILITY}

Reports produced after January 1, 1996, are generally available free via US Department of Energy (DOE) SciTech Connect.

Website osti.gov

Reports produced before January 1, 1996, may be purchased by members of the public from the following source:

National Technical Information Service

5285 Port Royal Road

Springfield, VA 22161

Telephone 703-605-6000 (1-800-553-6847)

TDD 703-487-4639

Fax 703-605-6900

E-mail info@ntis.gov

Website classic.ntis.gov

Reports are available to DOE employees, DOE contractors, Energy Technology Data Exchange representatives, and International Nuclear Information System representatives from the following source:

Office of Scientific and Technical Information

PO Box 62

Oak Ridge, TN 37831

Telephone 865-576-8401

Fax 865-576-5728

E-mail reports@osti.gov

Website osti.gov/contact

This report was prepared as an account of work sponsored by an agency of the United States Government. Neither the United States Government nor any agency thereof, nor any of their employees, makes any warranty, express or implied, or assumes any legal liability or responsibility for the accuracy, completeness, or usefulness of any information, apparatus, product, or process disclosed, or represents that its use would not infringe privately owned rights. Reference herein to any specific commercial product, process, or service by trade name, trademark, manufacturer, or otherwise, does not necessarily constitute or imply its endorsement, recommendation, or favoring by the United States Government or any agency thereof. The views and opinions of authors expressed herein do not necessarily state or reflect those of the United States Government or any agency thereof. 
ORNL/TM-2021/2056

Nuclear Energy and Fuel Cycle Division

\title{
IMPLEMENTATION OF THE WINDOWED MULTIPOLE METHOD IN SHIFT
}

\author{
Elliott D. Biondo
}

Date Published: July 15, 2021

\author{
Prepared by \\ OAK RIDGE NATIONAL LABORATORY \\ Oak Ridge, TN 37831-6283 \\ managed by \\ UT-Battelle, LLC \\ for the \\ US DEPARTMENT OF ENERGY \\ under contract DE-AC05-00OR22725
}




\section{CONTENTS}

LIST OF FIGURES $\ldots \ldots \ldots \ldots \ldots \ldots \ldots \ldots \ldots \ldots \ldots$ iv

LIST OF TABLES $\ldots \ldots \ldots \ldots \ldots \ldots \ldots \ldots \ldots \ldots \ldots$

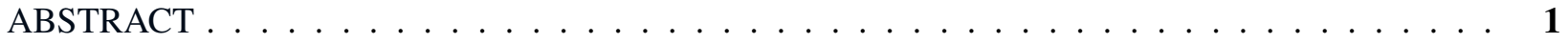

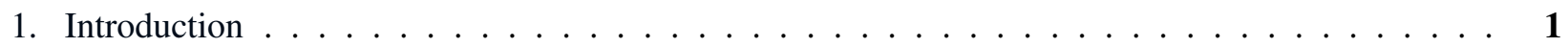

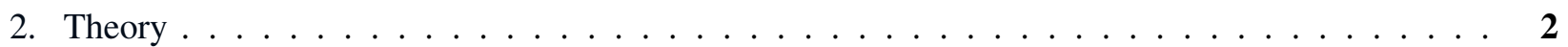

2.1 Original Multipole Method (Non-Windowed) $\ldots \ldots \ldots \ldots \ldots \ldots$

2.2 Windowed Multipole Method . . . . . . . . . . . . . . . . . . . . 3

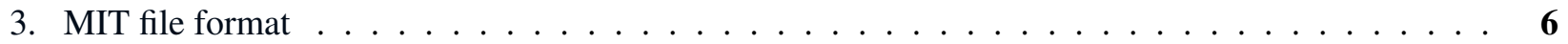

3.1 Bound vs. Unbound Hydrogen $\ldots \ldots \ldots \ldots$

4. Implementation and Validation . . . . . . . . . . . . . . . . . . . . 9

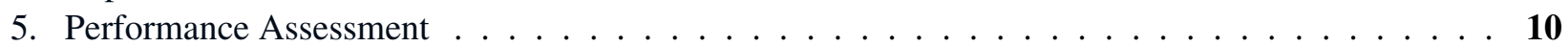

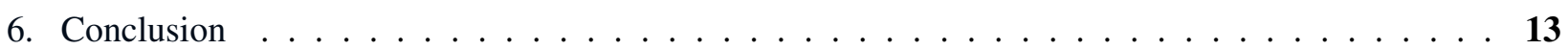

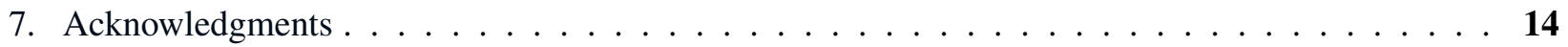

A. Shift Validation Results . . . . . . . . . . . . . . . . . . . . . . A-1 


\section{LIST OF FIGURES}

1 Quarter-core SMR model [3] used for tracking rate assessment. . . . . . . . . . . . . . 10

2 Tracking rate results for the fresh fuel case. . . . . . . . . . . . . . . 11

3 Tracking rate results for the depleted fuel case. . . . . . . . . . . . . . 11

4 The callgrind graph for the CPU version fresh core SMR problem. . . . . . . . . . . 12

5 Summary of callgrind results for the fresh core SMR problem. . . . . . . . . . . . . 12 


\section{LIST OF TABLES}

1 MIT file format parameters (Note: this is the presumed format of files that are read by Shift; it does not reflect how data are stored internally in Shift $) \ldots \ldots$. . . . . . . . . 7

2 Curvefit polynomial parameters produced by OpenMC and Shift . . . . . . . . . . . . 9

3 Maximum relative difference between Shift results and OpenMC results over all . . . . . . A-2 


\begin{abstract}
The windowed multipole (WMP) method has been implemented in the Shift Monte Carlo (MC) radiation transport code with support for both CPU and GPU execution. With this method, small WMP data libraries ( $100 \mathrm{MB})$ can be used to accurately Doppler broaden cross sections to arbitrary temperatures "on the fly" during an MC simulation. This approach yields significant memory savings relative to traditional methods, making it ideal for high-fidelity analysis such as coupled multiphysics simulations.

This document provides the exact forms of the WMP equations used by Shift, as well as a detailed description of the structure of WMP HDF5 data files provided by the Massachusetts Institute of Technology (MIT). The Shift implementation has been validated against the OpenMC radiation transport code, with excellent agreement demonstrated for 70 nuclides across an operative range of temperatures. CPU and GPU performance testing using a small module reactor (SMR) problem demonstrated that this method decreases the neutron tracking rate by a factor of $\sim 2$ on the Summitdev machine. A new set of WMP data being developed in-house will employ novel methods to improve tracking rates.
\end{abstract}




\section{INTRODUCTION}

Properly accounting for the temperature dependence of neutron cross sections is essential for criticality safety and multiphysics coupling applications. Neutron cross sections describe the probability per unit length that an incident neutron at energy $E$ will interact with a target nucleus within some medium with a bulk temperature $T$. This probability is dependent on the relative speed of the interaction, i.e., the magnitude of the difference between the incident neutron velocity vector and target nucleus velocity vector. Target speeds follow a Maxwellian distribution, centered around $T$. At higher $T$, target speeds are more widely distributed, leading to a wider distribution of possible relative speeds. As a result, at higher temperatures, neutron cross section resonances become shorter and wider, a phenomenon known as Doppler broadening. This effect is especially important in the resolved resonance region (RRR), where the magnitude of cross sections can vary by an order of magnitude or more over small energy intervals $(\sim 1 \mathrm{eV})$.

For Monte Carlo (MC) neutron transport simulations, Doppler broadening is traditionally accounted for by creating separate sets of point-wise "continuous energy" (CE) cross section libraries for each temperature within a temperature grid, using the SIGMA1 [1] algorithm for Doppler broadening. Temperature-dependent cross sections are then calculated by either looking up the cross section at the nearest temperature grid point or linearly interpolating between cross sections at two temperature grid points. When calculations require a fine temperature grid to accurately capture temperature effects, cross section storage requirements on the order of $\sim 1$ GB per temperature point make this method impractical.

The windowed multipole (WMP) method [5] can be used to calculate cross sections at any temperature "on the fly" during a Monte Carlo simulation. This can be accomplished from a single set of nuclear data on the order of $\sim 100 \mathrm{MB}$. This method calculates cross sections using pole and residue parameters from an alternative, mathematically equivalent nuclear data representation [4], and it has previously been implemented in the OpenMC [11] Monte Carlo radiation transport code.

The WMP method has been implemented on both the CPU and GPU sides of Shift MC radiation transport code [10], to be used with a set of WMP data generated by the Massachusetts Institute of Technology (MIT) Computational Reactor Physics Group. The remainder of this document is organized as follows. Section 2 describes the original non-windowed multipole method, followed by the WMP and the final forms of the WMP equation used in Shift. Section 3 describes the format of the HDF5-based WMP files provided by MIT. Section 4 describes the implementation of the WMP method in Shift and subsequent validation against OpenMC. Section 5 describes performance testing of the CPU and GPU implementation using a small modular reactor (SMR) problem. Section 6 provides concluding remarks. 


\section{THEORY}

In this section, the original non-windowed version of the multipole method is first introduced. The windowed multipole method is then described, and the final answers used in the Shift implementation are presented.

\subsection{ORIGINAL MULTIPOLE METHOD (NON-WINDOWED)}

The multipole method, originally proposed by Hwang [4], is based on the observation that the standard Rmatrix [12] form of the nuclear cross section can be reasonably approximated as a rational function in $\sqrt{(} E)$ space $^{1}$. A partial fraction expansion can be performed on this rational function using the residue method [8]. This provides an expression for the cross section in terms of poles - singularities in the complex plane - and residues, which are proportional to the path integrals around these singularities. For some reaction $x$, the $0 \mathrm{~K}$ cross section is

$$
\sigma_{x}(E, 0 \mathrm{~K})=\frac{1}{E} \sum_{j} \mathfrak{R}\left[\frac{-i r_{x, j}}{p_{j}-\sqrt{E}}\right],
$$

as seen in Forget et al. equation 4 [2]. In this equation $p_{j}$ are the poles, $r_{x, j}$ are reaction-specific residues, $i$ is the imaginary unit, and $\mathfrak{R}$ provides the real part of a complex-valued argument. To calculate the total cross section, two additional quantities are required: $\sigma_{p}(E)$, the potential cross section; and $e^{-2 i \varphi_{l}}$, which is referred to as the "total cross section factor," where $\varphi_{l}$ is the hard-sphere phase shift for the angular momentum quantum number $l$, noting that each resonance has an associated $l$. The total cross section is given by

$$
\sigma_{t}(E, 0 \mathrm{~K})=\sigma_{p}(E)+\frac{1}{E} \sum_{j} \mathfrak{R}\left[e^{-2 i \varphi_{l}} \frac{-i r_{x, j}}{p_{j}-\sqrt{E}}\right] \text {, }
$$

as seen in Forget et al. equation 5. In Josey et al. [5], a slightly different but equivalent formulation is used with the $-i$ omitted. This document uses the Forget et al. formulation. The $\varphi_{l}$ is calculated using the recursive relationship

$$
\varphi_{l}=\varphi_{l-1}-\tan ^{-1}\left(\frac{P_{l-1}}{l-S_{l-1}}\right), \quad \varphi_{0}=\rho_{l}
$$

where the penetration factor is given by

$$
P_{l}=\frac{\rho_{l}^{2} P_{l-1}}{\left(l-S_{l-1}\right)^{2}+P_{l-1}^{2}}, \quad P_{0}=\rho_{l},
$$

and the shift factor is given by

$$
S_{l}=\frac{\rho_{l}^{2}\left(l-S_{l-1}\right)}{\left(l-S_{l-1}\right)^{2}+P_{l-1}^{2}}-l, \quad S_{0}=0 .
$$

\footnotetext{
${ }^{1} \mathrm{~A}$ rational function is one that can be expressed as the ratio of two polynomials.
} 
Values of $\varphi_{l}$ for $l=[0,3]$ are

$$
\varphi_{l}= \begin{cases}\rho_{0}, & l=0, \\ \rho_{1}-\tan ^{-1}\left(\rho_{1}\right), & l=1, \\ \rho_{2}-\tan ^{-1}\left(\frac{3 \rho_{2}}{3-\rho_{2}^{2}}\right), & l=2, \\ \rho_{3}-\tan ^{-1}\left(\frac{\rho_{3}\left(15-\rho_{3}^{2}\right)}{15-6 \rho_{3}^{2}}\right), & l=3,\end{cases}
$$

as seen in the SAMMY manual [6]. The quantity $\rho$ must be provided by the WMP data.

These cross sections can be Doppler broadened to temperature $T$ using the following equations:

$$
\begin{gathered}
\sigma_{x}(E, \xi)=\frac{1}{2 E \sqrt{\xi}} \sum_{j} \mathfrak{R}\left[r_{x, j} \sqrt{\pi} W\left(z_{0, j}\right)-\frac{-i r_{x, j}}{\sqrt{\pi}} C\left(\frac{p_{j}}{\sqrt{\xi}}, \frac{\sqrt{E}}{2 \sqrt{\xi}}\right)\right], \\
\sigma_{t}(E, \xi)=\sigma_{p}(E)+\frac{1}{2 E \sqrt{\xi}} \sum_{j} \mathfrak{R}\left[e^{-2 i \varphi_{l}}\left[r_{x, j} \sqrt{\pi} W\left(z_{0, j}\right)-\frac{-i r_{x, j}}{\sqrt{\pi}} C\left(\frac{p_{j}}{\sqrt{\xi}}, \frac{\sqrt{E}}{2 \sqrt{\xi}}\right)\right]\right],
\end{gathered}
$$

with

$$
\xi=\frac{k T}{4 A}
$$

and

$$
z_{0, j}=\frac{\sqrt{E}-p_{j}}{2 \sqrt{\xi}}
$$

as seen in Forget et al. Equations 7, 8, and 9. In these equations, $W$ is the Faddeeva function. The function $C$ can be neglected for "most practical cases" [4] and is therefore excluded henceforth.

\subsection{WINDOWED MULTIPOLE METHOD}

Using the original multipole method described in Section 2.1, the Faddeeva function must be evaluated for each pole. This approach is prohibitively computationally expensive, as important nuclides such as ${ }^{238} \mathrm{U}$ have $O\left(10^{3}\right)$ poles. With the windowed multipole method, the summations in Equations 7 and 8 are truncated so that the Faddeeva function is only evaluated for the subset of the poles. The poles outside of this subset are accounted for using an approximation.

With this approach, energy-space is subdivided into a series of non-overlapping "inner windows" spaced uniformly in $\sqrt{E}$-space. For each inner window, an outer window is created which contains all of the poles that significantly contribute to the cross section within the inner window. For some energy $E$ within the inner window, the cross section is calculated in terms of contributions from poles inside the outer window, and outside of the outer window:

$$
\sigma=\sigma_{\text {inside }}+\sigma_{\text {outside }}
$$


The $\sigma_{\text {inside }}$ is calculated using the equations provided in Section 2.1, with summation indices from $w_{\text {start }}$ to $w_{\text {end }}$. The contributions from poles outside the outer window are treated using a polynomial fitting function with $N$ terms in the form, as adapted from Josey et al., Equation 14.

$$
\sigma_{\text {outside }}(E, 0 \mathrm{~K})=\sum_{n=0}^{N-1} a_{w, n} E^{(n-2) / 2},
$$

where values of $a_{w, n}$ are specific to reactions. In order to Doppler broaden $\sigma_{\text {outside }}$, the same $a_{w, n}$ values are used in the equation

$$
\sigma_{\text {outside }}(E, \xi)=\sum_{n=0}^{N-1} a_{w, n} \mathfrak{D}_{n}
$$

where

$$
\mathfrak{D}_{n}= \begin{cases}\frac{1}{E} \operatorname{erf}\left(\frac{\sqrt{E}}{2 \sqrt{\xi}}\right), & n=0, \\ \frac{1}{\sqrt{E}}, & n=1, \\ (2 \xi+E) \frac{1}{E} \operatorname{erf}\left(\frac{\sqrt{E}}{2 \sqrt{\xi}}\right)+2 \sqrt{\frac{\xi}{\pi E}} \mathrm{e}^{-\frac{E}{4 \xi}}, & n=2, \\ {[2 \xi(2 n-3)+E] \mathfrak{D}_{n-2},} & n=3, \\ {[2 \xi(2 n-3)+E] \mathfrak{D}_{n-2}-(2 \xi)^{2}(n-2)(n-3) \mathfrak{D}_{n-4},} & n \geq 4 .\end{cases}
$$

as adapted from Josey et al., Equations 15 and 16.

\subsubsection{FINAL ANSWERS}

The final answers for the $0 \mathrm{~K}$ cross sections can be found by combining the expressions for the cross sections inside and outside the window:

$$
\begin{gathered}
\sigma_{x}(E, 0 \mathrm{~K})=\frac{1}{E} \sum_{j=w_{\min }}^{w_{\max }} \mathfrak{R}\left[\frac{-i r_{x, j}}{p_{j}-\sqrt{E}}\right]+\sum_{n=0}^{N-1} a_{x, w, n} E^{(n-2) / 2}, \\
\sigma_{t}(E, 0 \mathrm{~K})=\frac{1}{E} \sum_{j=w_{\min }}^{w_{\text {max }}} \mathfrak{R}\left[e^{-2 i \varphi_{l}} \frac{-i r_{x, j}}{p_{j}-\sqrt{E}}\right]+\sum_{n=0}^{N-1} a_{t, w, n} E^{(n-2) / 2} .
\end{gathered}
$$

The final answers for the Doppler broadened cross sections are as follows:

$$
\begin{gathered}
\sigma_{x}(E, \xi)=\frac{1}{2 E \sqrt{\xi}} \sum_{j=w_{\min }}^{w_{\max }} \mathfrak{R}\left[\sqrt{\pi} r_{x, j} W\left(z_{0, j}\right)\right]+\sum_{n=0}^{N-1} a_{x, w, n} \mathfrak{D}_{n}, \\
\sigma_{t}(E, \xi)=\frac{1}{2 E \sqrt{\xi}} \sum_{j=w_{\min }}^{w_{\max }} \mathfrak{R}\left[e^{-2 i \varphi_{l}}\left[\sqrt{\pi} r_{x, j} W\left(z_{0, j}\right)\right]\right]+\sum_{n=0}^{N-1} a_{t, w, n} \mathfrak{D}_{n} .
\end{gathered}
$$

The potential cross section $\sigma_{p}$, which appears in Equation 8 is accounted for by the fitting parameters [7]. 
Up to this point, it has been assumed that poles and residues use the Reich Moore (RM) formalism. In some cases, the nuclear data available necessitates the use of the Multi-Level Breit-Wigner (MLBW) formalism. When using MLBW poles and residues, an additional residue, $r_{c}$, is necessary to represent the "competitive" cross section. This affects the calculation of the total cross section, as shown below:

$$
\begin{gathered}
\sigma_{t}(E, 0 \mathrm{~K})=\frac{1}{E} \sum_{j=w_{\min }}^{w_{\max }} \mathfrak{R}\left[e^{-2 i \varphi_{l}} \frac{-i\left(r_{x, j}+r_{c, j}\right)}{p_{j}-\sqrt{E}}\right]+\sum_{n=0}^{N-1} a_{t, w, n} E^{(n-2) / 2} . \\
\sigma_{t}(E, \xi)=\frac{1}{2 E \sqrt{\xi}} \sum_{j=w_{\min }}^{w_{\max }} \mathfrak{R}\left[\sqrt{\pi}\left[e^{-2 i \varphi_{l}} r_{x, j}+r_{c, j}\right] W\left(z_{0, j}\right)\right]+\sum_{n=0}^{N-1} a_{t, w, n} \mathfrak{D}_{n} .
\end{gathered}
$$




\section{MIT FILE FORMAT}

The equations used to calculate the cross section described in Section 2 require custom nuclear data libraries containing poles, corresponding residues, and other parameters. In January 2018, a WMP library containing 71 nuclides in separate HDF5 files was provided by the MIT Computational Reactor Physics Group. Subsequently, the complete version of this library, containing 423 nuclides, was provided by email in April, 2018. The total size of these 423 HDF5 files on disk is 91 MB. The format of these files is described below. Subsequent versions of this library are publicly available on GitHub [9], but contain files in a slightly different format.

Data for each nuclide was supplied in files named as ZZZAAA. h5, where ZZZ and AAA always have 3 digits. Each of these files is independent and contains all data necessary to calculate cross sections using the WMP method. Some of the datasets in these HDF5 files are extraneous, and others store necessary data in an idiosyncratic way. Table 1 lists only the datasets that are read from these HDF5 files by Shift. Note that the descriptions only apply to the MIT file format; they do not describe how data are actually stored in Shift.

To use the MIT-supplied data, all of the individual HDF5 files must be linked into a single HDF5 file using external links. This can be done using the code below. The resulting single file can be specified in the Omnibus using the ce_pole_data_path parameter.

\section{Listing 1. Python code for creating a top-level HDF5 file.}

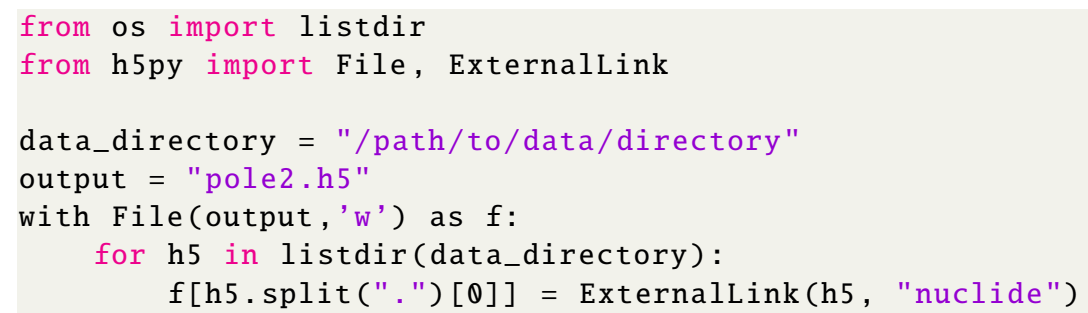


Table 1. MIT file format parameters (Note: this is the presumed format of files that are read by Shift; it does not reflect how data are stored internally in Shift)

\begin{tabular}{|c|c|c|}
\hline dataset(s) & type & description \\
\hline num_1 & int & The number of possible $l$ quantum states \\
\hline fissionable & bool & Whether or not the nuclide is fissionable \\
\hline formalism & int & Either 2 for MLBW or 3 for RM \\
\hline spacing & double & The width of the windows in $\sqrt{E}$-space \\
\hline start_E, end_E & double & $\begin{array}{l}\text { The minimum and maximum incident neutron en- } \\
\text { ergies for which the data is valid }\end{array}$ \\
\hline pseudo_k@RS & double & $\begin{array}{l}\text { Equivalent to } \rho / \sqrt{E} \text {, an intermediate quantity used } \\
\text { to calculate } \phi_{l} \text {, }\end{array}$ \\
\hline sqrt_AWR & double & The square root of the atomic weight ratio \\
\hline length & int & The number of poles \\
\hline windows & int & The number of windows \\
\hline fit_order & int & $\begin{array}{l}\text { Add } 1 \text { to this field to get the number of terms in } \\
\text { the polynomial }\end{array}$ \\
\hline 1_value & $1 \mathrm{D}$ array of int & $\begin{array}{l}\text { Subtract } 1 \text { from each value in the array to get the } l \\
\text { quantum number for each pole }\end{array}$ \\
\hline w_start, w_end & $1 \mathrm{D}$ array of int & $\begin{array}{l}\text { The indices of the first and last poles (inclusive) } \\
\text { included in each window, indexed from } 1\end{array}$ \\
\hline broaden_poly & 1D array of bool & $\begin{array}{l}\text { Whether or not to broaden the polynomial within } \\
\text { each window }\end{array}$ \\
\hline data & 2D array of complex $<$ double $>$ & $\begin{array}{l}\text { The pole and reside data. The first dimension is } \\
\text { the pole index. The second dimension provides } \\
\text { the poles and the residues. For RM formalism, } \\
\text { the order the data appears in the second dimension } \\
\text { is pole, total residue, absorption residue, fission } \\
\text { residue (when applicable). For MLBW formal- } \\
\text { ism, the order is pole, total residue, competitive } \\
\text { residue, absorption residue, fission residue (when } \\
\text { applicable). }\end{array}$ \\
\hline curvefit & 3D array complex $<$ double $>$ & $\begin{array}{l}\text { The polynomial data. The first dimension is the } \\
\text { window index, the second dimension is the reac- } \\
\text { tion index (appearing in the same order as data), } \\
\text { and the third dimension is the polynomial index. }\end{array}$ \\
\hline
\end{tabular}




\subsection{BOUND VS. UNBOUND HYDROGEN}

The MIT WMP library contains a single file for ${ }^{1} \mathrm{H}$. This file, $001001 . \mathrm{h} 5$, contains data for unbound ${ }^{1} H$. This was initially a source of confusion, as in SCALE "1001" denotes bound hydrogen, and "8001001" denotes unbound hydrogen. Furthermore, pole data is not valid for bound nuclides in the $S(\alpha, \beta)$ region. To remedy this, the 001001 . h5 file was modified to represent bound hydrogen by moving the lower energy bound (i.e., start_E) to be the lower energy bound of the second window, well above the $S(\alpha, \beta)$ region. This was done using the script below.

\section{Listing 2. Python code for changing the lower bound of the 1001 data.}

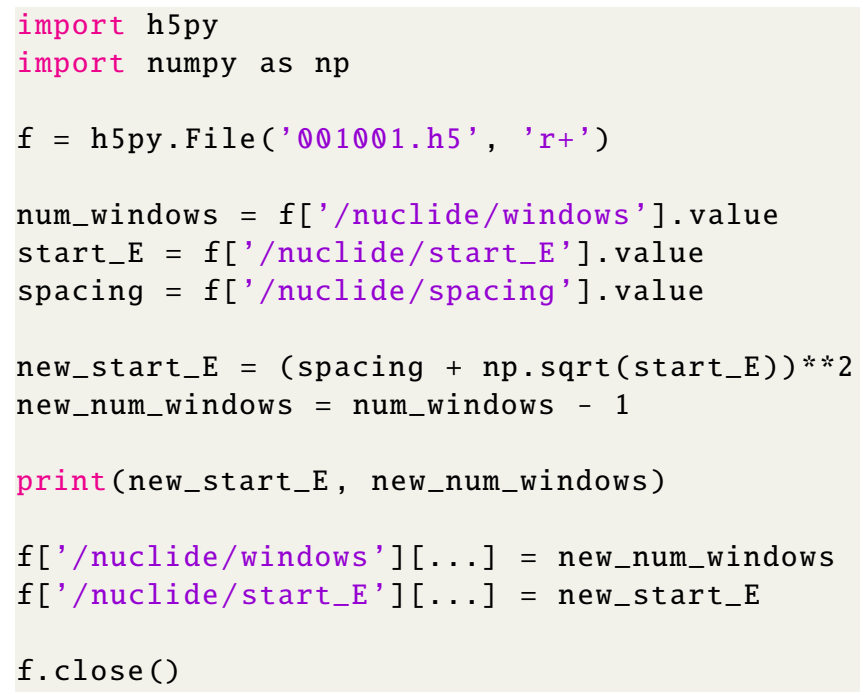




\section{IMPLEMENTATION AND VALIDATION}

The WMP method was implemented in Shift by creating three main classes_- Pole_Reader, Pole_Data, and Pole_XS_Calculator-with the latter two implemented on both the CPU and the GPU. The Pole_Reader class reads the top-level HDF5 file of the MIT WMP data and stores pertinent information in Pole_Data objects (one Pole_Data object per nuclide). The Pole_XS_Calculator class calculates the cross section at a given energy and temperature using Pole_Data objects.

Although the functionality added to Shift is unit tested, additional validation was performed by comparing cross sections produced using the WMP method in Shift to those produced using the WMP method in OpenMC. For 70 of the 71 original nuclides, cross sections were calculated with Shift and OpenMC at each energy point in the continuous energy data between start_E and end_E. The 001001 .h5 file was not included in this analysis because of the (now resolved) issue described in Section 3.1. The maximum relative difference between the results was then calculated using the formula

$$
\text { maximum relative difference }=\max _{\left[E_{\min }, E_{\max }\right]}\left(\frac{\left|\sigma_{\text {Shift }}(E)-\sigma_{\text {OpenMC }}(E)\right|}{\sigma_{\text {OpenMC }}(E)}\right) .
$$

This was done for all applicable reactions at three different temperatures: $T=0 \mathrm{~K}, T=300 \mathrm{~K}$, and $T=1000 \mathrm{~K}$. These results appear in Appendix A. For the majority of the combinations of nuclide, reaction, and temperature, the maximum relative difference was less than $1 \times 10^{-6}$. The one notable outlier was ${ }^{52} \mathrm{Cr}(\mathrm{ZAID}=24052)$. Maximum relative differences of $2.18 \times 10^{-3}$ and $2.64 \times 10^{-3}$ were observed for the absorption cross sections at $T=300 \mathrm{~K}$ and $T=1000 \mathrm{~K}$, respectively.

Further investigation was conducted to determine the source of the discrepancy in the $T=300 \mathrm{~K}$ case. It was found that this difference occurs at an energy of $572104.75001 \mathrm{eV}$, where Shift calculates the cross section to be $5.42641 \times 10^{-4} \mathrm{~b}$, and OpenMC calculates the cross section to be $5.41210 \times 10^{-4} \mathrm{~b}$. This energy is within a window where no poles are present and broaden_poly is true, meaning that cross section comes entirely from the contribution of the polynomial via Equation 13. The $a_{n}$ and $\mathfrak{D}_{n}$ terms in this equation, as calculated by OpenMC and Shift, are shown in Table 2. These values are all identical within 12 decimal places, which indicates that the discrepancy in the cross section is a floating-point artifact that arises from Equation 13, which involves the addition and subtraction of values that vary over many orders of magnitude.

Table 2. Curvefit polynomial parameters produced by OpenMC and Shift

\begin{tabular}{|c|c|c|c|c|}
\hline \multirow[b]{2}{*}{$\mathrm{n}$} & \multicolumn{2}{|c|}{$a_{n}$} & \multicolumn{2}{|c|}{$\mathfrak{D}_{n}$} \\
\hline & OpenMC & Shift & OpenMC & Shift \\
\hline$\overline{0}$ & $3.563678816713 \times 10^{14}$ & $3.563678816713 \times 10^{14}$ & $\overline{1.747931650598 \times 10^{-6}}$ & $1.747931650598 \times 10^{-6}$ \\
\hline 1 & $-1.885377990642 \times 10^{12}$ & $-1.885377990642 \times 10^{12}$ & $1.322093661810 \times 10^{-3}$ & $1.322093661810 \times 10^{-3}$ \\
\hline 2 & $3.740499363623 \times 10^{9}$ & $3.740499363623 \times 10^{9}$ & $1.000000001463 \times 10^{0}$ & $1.000000001463 \times 10^{0}$ \\
\hline 3 & $-3.298209410335 \times 10^{6}$ & $-3.298209410335 \times 10^{6}$ & $7.563760671983 \times 10^{2}$ & $7.563760671983 \times 10^{2}$ \\
\hline 4 & $1.090581541091 \times 10^{3}$ & $1.090581541091 \times 10^{3}$ & $5.721047550304 \times 10^{5}$ & $5.721047550304 \times 10^{5}$ \\
\hline
\end{tabular}




\section{PERFORMANCE ASSESSMENT}

The CPU and GPU implementations of the WMP method in Shift were performance tested by measuring neutron tracking rates during $k$-eigenvalue calculations of a quarter-core reactor model based on the NuScale SMR [3], as shown in Figure 1. Simulations were run using both fresh and depleted fuels. For the fresh fuel case, a single fuel material was used, consisting of 5 nuclides. The depleted fuel case consists of the fresh fuel material depleted for 30 days with 5-day time steps, resulting in 12,570 unique fuel materials containing an average of 240 nuclides.

All transport was performed at $595 \mathrm{~K}$, corresponding to a SCALE CE library temperature. This provides the most conservative timing comparison, as it is the best scenario for CE data, and only a single temperature library needs to be loaded. Trials were run on a single node of the Summitdev machine consisting of 2 IBM Power8+ CPUs and 4 NVIDIA Tesla P100 GPUs. Results are are shown in Figures 2 and 3.

These results show a consistent factor of $\sim 2$ decrease in the tracking rate when using the WMP method on both the CPU and the GPU. This was investigated using callgrind on the CPU with the fresh fuel case to identify opportunities for future improvements. The resultant callgrind graph is shown in Figure 4 and is summarized Figure 5.

The results in Figure 5 show that $36 \%$ of execution time is spent evaluating the Faddeeva function and math built-ins. Work is currently underway to mitigate this issue. Reasonable approximations can be made to eliminate error function evaluations from the broadened polynomial. Improved windowing schemes will reduce the average and maximum number of poles per window, which should reduce the number of Faddeeva evaluations per cross section calculation. Progress on this forthcoming WMP library will be reported in a future document.

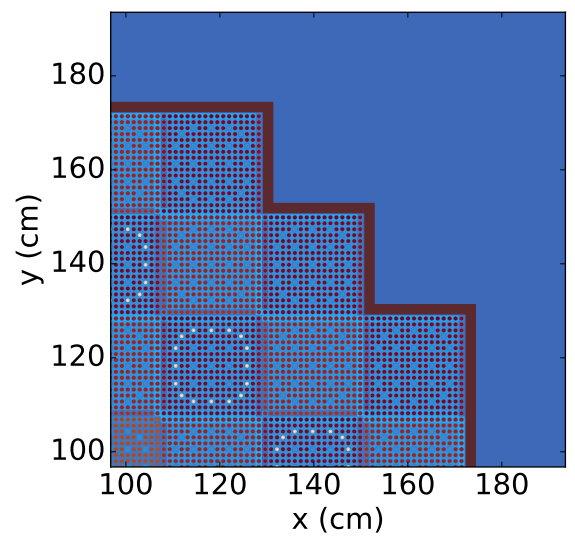

Figure 1. Quarter-core SMR model [3] used for tracking rate assessment. 


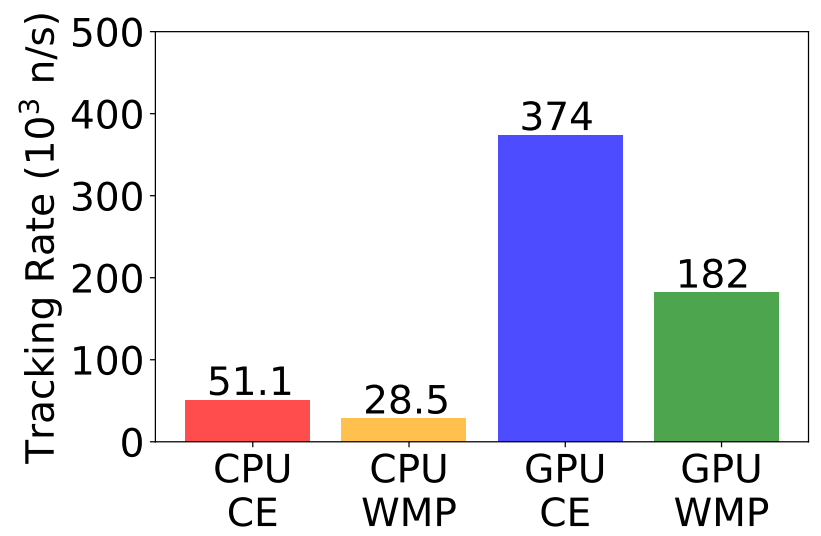

Figure 2. Tracking rate results for the fresh fuel case.

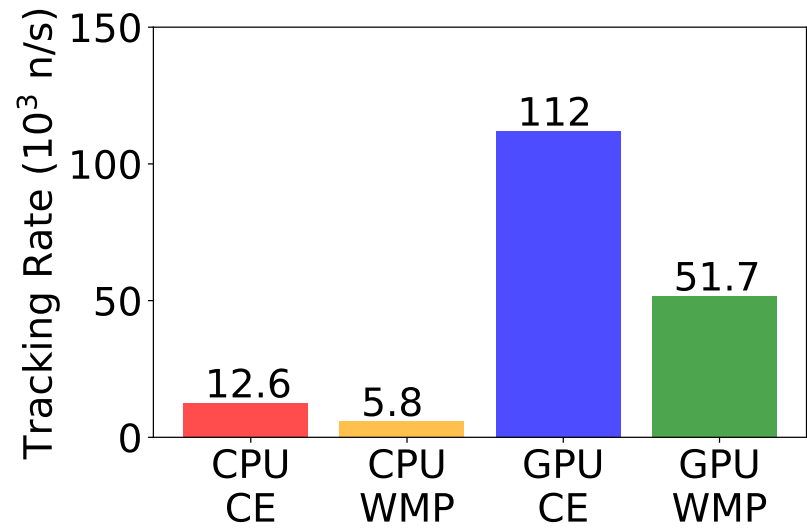

Figure 3. Tracking rate results for the depleted fuel case. 


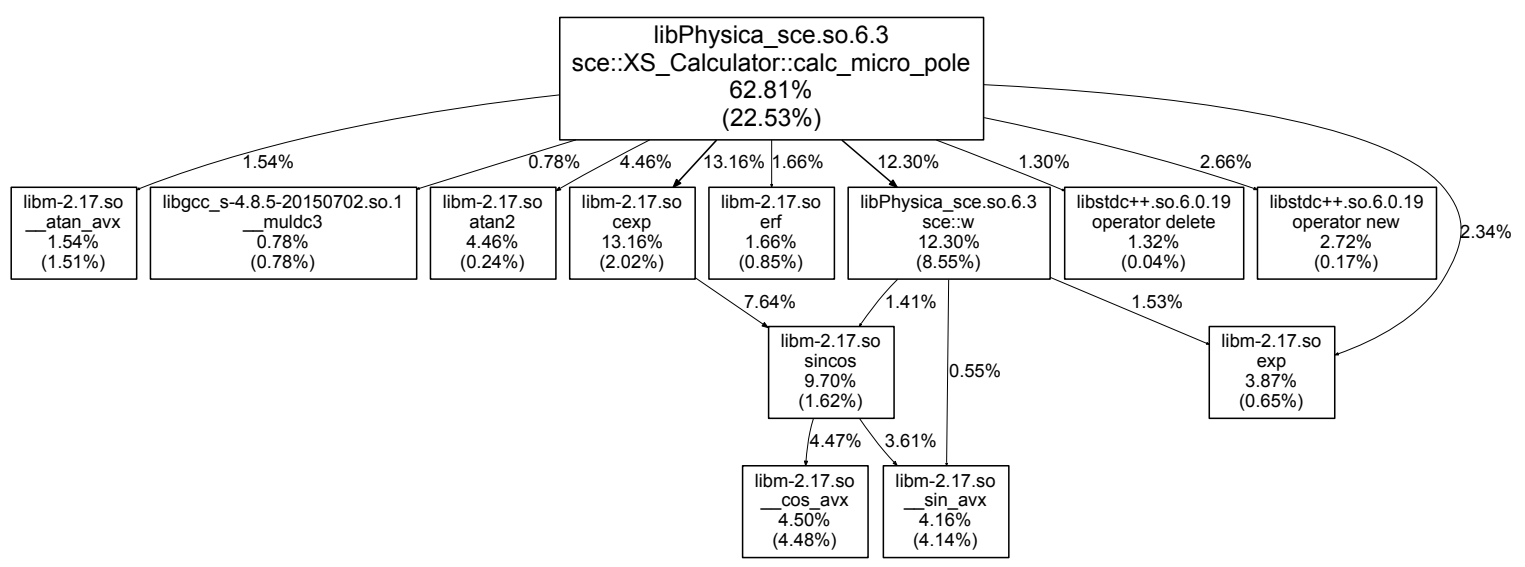

Figure 4. The callgrind graph for the CPU version fresh core SMR problem.

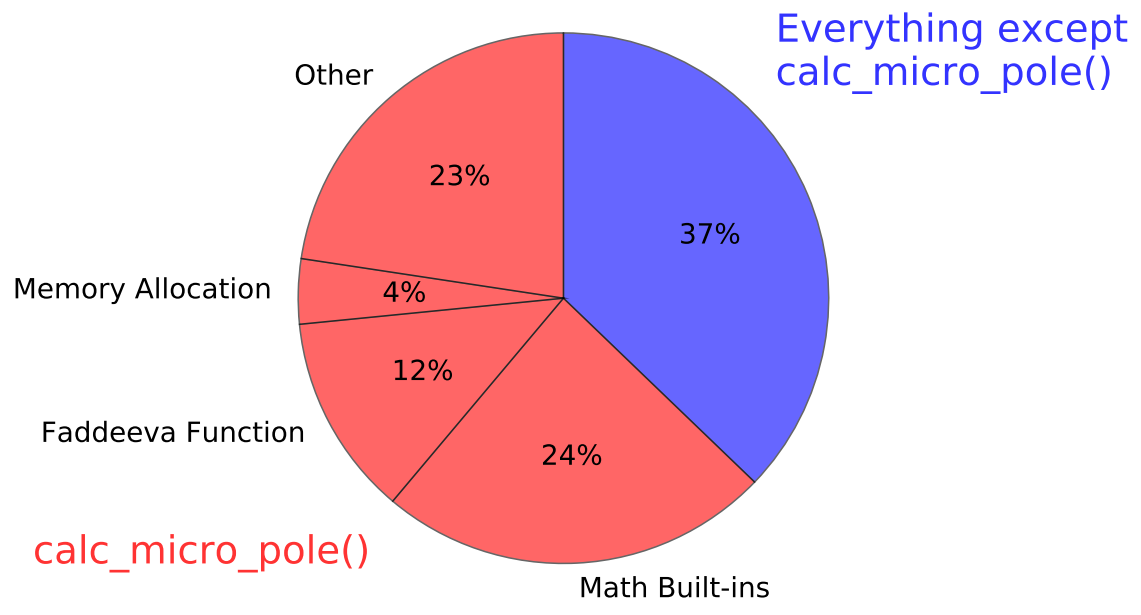

Figure 5. Summary of callgrind results for the fresh core SMR problem. 


\section{CONCLUSION}

The windowed multipole method has been implemented on the CPU and GPU sides of Shift, allowing for accurate Doppler broadening at arbitrary temperatures with less than $100 \mathrm{MB}$ of RAM. This memory savings comes with a trade-off: the factor of $\sim 2$ reduction in the CPU and GPU particle tracking rate. When combined with the complete 423-nuclide WMP data library supplied by MIT, this capability is ready for production-level use. Future work is currently underway to generate WMP data in-house in order to create data sets that will not incur the same performance penalties. 


\section{ACKNOWLEDGMENTS}

This research was supported by the Exascale Computing Project (ECP), Project Number: 17-SC-20-SC, a collaborative effort of two DOE organizations: the Office of Science and the National Nuclear Security Administration. These offices are responsible for the planning and preparation of a capable exascale ecosystem-including software, applications, hardware, advanced system engineering, and early testbed platforms - to support the nation's exascale computing imperative.

This work was supported by Oak Ridge National Laboratory, which is managed and operated by UT-Battelle, LLC, for the US Department of Energy under Contract No. DEAC05-00OR22725. This research used resources of the Oak Ridge Leadership Computing Facility at Oak Ridge National Laboratory, which is supported by the Office of Science of the US Department of Energy under Contract No. DE-AC05-00OR22725. 


\section{REFERENCES}

[1] D. E. Cullen and C. R. Weisbin. Exact Doppler broadening of tabulated cross sections. Nuclear Science and Engineering, 60(3):199-229, 1976.

[2] B. Forget, S. Xu, and K. Smith. Direct Doppler broadening in Monte Carlo simulations using the multipole representation. Annals of Nuclear Energy, 64:78 - 85, 2014.

[3] S. P. Hamilton and T. M. Evans. Continuous-energy Monte Carlo neutron transport on GPUs in the Shift code. Annals of Nuclear Energy, 128:236 - 247, 2019.

[4] R. N. Hwang. A rigorous pole representation of multilevel cross sections and its practical applications. Nuclear Science and Engineering, 96(3):192-209, 1987.

[5] C. Josey, P. Ducru, B. Forget, and K. Smith. Windowed multipole for cross section Doppler broadening. Journal of Computational Physics, 307:715 - 727, 2016.

[6] N. M. Larson. Updated users' guide for SAMMY: Multilevel R-matrix fits to neutron data using Bayes' equations. Technical Report ORNL/TM-9179/R8, Oak Ridge National Laboratory, 2008.

[7] J. Liang. Private Communication, February 2018.

[8] MathWorks. Partial fraction expansion (partial fraction decomposition), https://www . mathworks . com/help/matlab/ref/residue.html.

[9] MIT Computational Reactor Physics Group. Windowed multipole library. https://github.com/ mit-crpg/WMP_Library.

[10] T. M. Pandya, S. R. Johnson, T. M. Evans, G. G. Davidson, S. P. Hamilton, and A. T. Godfrey. Implementation, capabilities, and benchmarking of Shift, a massively parallel Monte Carlo radiation transport code. Journal of Computational Physics, 308:239-272, Mar. 2016.

[11] P. K. Romano and B. Forget. The OpenMC Monte Carlo particle transport code. Annals of Nuclear Energy, 51:274, 2013.

[12] E. P. Wigner and L. Eisenbud. Higher angular momenta and long range interaction in resonance reactions. Phys. Rev., 72:29-41, Jul 1947. 
APPENDIX A. SHIFT VALIDATION RESULTS 


\section{APPENDIX A. SHIFT VALIDATION RESULTS}

Table 3. Maximum relative difference between Shift results and OpenMC results over all

\begin{tabular}{|c|c|c|c|c|c|c|c|c|c|}
\hline \multirow[b]{2}{*}{ ZAID } & \multicolumn{3}{|c|}{$T=0 \mathrm{~K}$} & \multicolumn{3}{|c|}{$T=300 \mathrm{~K}$} & \multicolumn{3}{|c|}{$T=1000 \mathrm{~K}$} \\
\hline & total & absorption & fission & total & absorption & fission & total & absorption & fission \\
\hline 13027 & $5.82 \times 10^{-8}$ & $5.92 \times 10^{-8}$ & - & $2.11 \times 10^{-7}$ & $4.44 \times 10^{-7}$ & - & $1.65 \times 10^{-7}$ & $4.86 \times 10^{-7}$ & - \\
\hline 14028 & $5.90 \times 10^{-8}$ & $5.85 \times 10^{-8}$ & - & $1.59 \times 10^{-7}$ & $7.95 \times 10^{-7}$ & - & $1.60 \times 10^{-7}$ & $9.03 \times 10^{-7}$ & - \\
\hline 14029 & $5.77 \times 10^{-8}$ & $5.81 \times 10^{-8}$ & - & $1.49 \times 10^{-7}$ & $1.77 \times 10^{-7}$ & - & $1.63 \times 10^{-7}$ & $2.54 \times 10^{-7}$ & - \\
\hline 14030 & $5.93 \times 10^{-8}$ & $5.87 \times 10^{-8}$ & - & $2.58 \times 10^{-7}$ & $2.95 \times 10^{-7}$ & - & $3.40 \times 10^{-7}$ & $4.08 \times 10^{-7}$ & - \\
\hline 16032 & $5.90 \times 10^{-8}$ & $5.89 \times 10^{-8}$ & - & $2.58 \times 10^{-7}$ & $5.38 \times 10^{-7}$ & - & $2.46 \times 10^{-7}$ & $6.90 \times 10^{-7}$ & - \\
\hline 16033 & $5.91 \times 10^{-8}$ & $5.90 \times 10^{-8}$ & - & $1.24 \times 10^{-7}$ & $8.20 \times 10^{-8}$ & - & $1.40 \times 10^{-7}$ & $1.20 \times 10^{-7}$ & - \\
\hline 16034 & $5.83 \times 10^{-8}$ & $5.72 \times 10^{-8}$ & - & $1.30 \times 10^{-7}$ & $5.80 \times 10^{-8}$ & - & $1.50 \times 10^{-7}$ & $5.87 \times 10^{-8}$ & - \\
\hline 20040 & $5.90 \times 10^{-8}$ & $5.91 \times 10^{-8}$ & - & $3.14 \times 10^{-7}$ & $8.68 \times 10^{-7}$ & - & $5.24 \times 10^{-7}$ & $9.41 \times 10^{-7}$ & - \\
\hline 20042 & $5.93 \times 10^{-8}$ & $5.91 \times 10^{-8}$ & - & $4.97 \times 10^{-7}$ & $4.84 \times 10^{-7}$ & - & $6.10 \times 10^{-7}$ & $5.61 \times 10^{-7}$ & - \\
\hline 20043 & $5.94 \times 10^{-8}$ & $5.88 \times 10^{-8}$ & - & $2.30 \times 10^{-7}$ & $3.44 \times 10^{-7}$ & - & $2.14 \times 10^{-7}$ & $4.58 \times 10^{-7}$ & - \\
\hline 20044 & $5.86 \times 10^{-8}$ & $5.85 \times 10^{-8}$ & - & $3.41 \times 10^{-7}$ & $5.27 \times 10^{-7}$ & - & $5.88 \times 10^{-7}$ & $6.68 \times 10^{-7}$ & - \\
\hline 20048 & $5.83 \times 10^{-8}$ & $5.71 \times 10^{-8}$ & - & $9.79 \times 10^{-8}$ & $5.86 \times 10^{-8}$ & - & $1.14 \times 10^{-7}$ & $6.32 \times 10^{-8}$ & - \\
\hline 22046 & $5.92 \times 10^{-8}$ & $5.94 \times 10^{-8}$ & - & $3.33 \times 10^{-7}$ & $6.70 \times 10^{-7}$ & - & $4.11 \times 10^{-7}$ & $7.68 \times 10^{-7}$ & - \\
\hline 22047 & $5.89 \times 10^{-8}$ & $5.86 \times 10^{-8}$ & - & $1.71 \times 10^{-7}$ & $5.24 \times 10^{-7}$ & - & $2.23 \times 10^{-7}$ & $5.59 \times 10^{-7}$ & - \\
\hline 22048 & $5.95 \times 10^{-8}$ & $5.90 \times 10^{-8}$ & - & $4.65 \times 10^{-7}$ & $8.83 \times 10^{-7}$ & - & $6.31 \times 10^{-7}$ & $8.43 \times 10^{-7}$ & - \\
\hline 22049 & $9.79 \times 10^{-8}$ & $1.09 \times 10^{-7}$ & - & $1.97 \times 10^{-7}$ & $6.03 \times 10^{-7}$ & - & $1.94 \times 10^{-7}$ & $6.96 \times 10^{-7}$ & - \\
\hline 22050 & $5.85 \times 10^{-8}$ & $5.90 \times 10^{-8}$ & - & $4.16 \times 10^{-7}$ & $8.07 \times 10^{-7}$ & - & $3.87 \times 10^{-7}$ & $8.59 \times 10^{-7}$ & - \\
\hline 23050 & $5.92 \times 10^{-8}$ & $9.49 \times 10^{-8}$ & - & $6.22 \times 10^{-8}$ & $1.43 \times 10^{-7}$ & - & $7.96 \times 10^{-8}$ & $1.61 \times 10^{-7}$ & - \\
\hline 23051 & $5.85 \times 10^{-8}$ & $5.89 \times 10^{-8}$ & - & $2.13 \times 10^{-7}$ & $5.36 \times 10^{-7}$ & - & $1.76 \times 10^{-7}$ & $5.70 \times 10^{-7}$ & - \\
\hline 24050 & $8.34 \times 10^{-6}$ & $6.23 \times 10^{-5}$ & - & $6.37 \times 10^{-6}$ & $6.23 \times 10^{-5}$ & - & $4.44 \times 10^{-6}$ & $6.22 \times 10^{-5}$ & - \\
\hline 24052 & $4.60 \times 10^{-5}$ & $9.97 \times 10^{-4}$ & - & $6.04 \times 10^{-5}$ & $2.18 \times 10^{-3}$ & - & $9.14 \times 10^{-5}$ & $2.64 \times 10^{-3}$ & - \\
\hline 24053 & $9.70 \times 10^{-6}$ & $9.73 \times 10^{-5}$ & - & $2.21 \times 10^{-5}$ & $9.73 \times 10^{-5}$ & - & $3.27 \times 10^{-5}$ & $9.73 \times 10^{-5}$ & - \\
\hline 24054 & $5.92 \times 10^{-8}$ & $5.93 \times 10^{-8}$ & - & $4.01 \times 10^{-7}$ & $8.00 \times 10^{-7}$ & - & $3.22 \times 10^{-7}$ & $9.35 \times 10^{-7}$ & - \\
\hline 25055 & $5.00 \times 10^{-7}$ & $7.97 \times 10^{-7}$ & - & $4.66 \times 10^{-7}$ & $8.19 \times 10^{-7}$ & - & $4.54 \times 10^{-7}$ & $8.54 \times 10^{-7}$ & - \\
\hline 26054 & $5.94 \times 10^{-8}$ & $5.91 \times 10^{-8}$ & - & $2.73 \times 10^{-7}$ & $5.27 \times 10^{-7}$ & - & $3.13 \times 10^{-7}$ & $6.29 \times 10^{-7}$ & - \\
\hline 26056 & $5.93 \times 10^{-8}$ & $5.93 \times 10^{-8}$ & - & $3.31 \times 10^{-7}$ & $7.74 \times 10^{-7}$ & - & $4.47 \times 10^{-7}$ & $7.99 \times 10^{-7}$ & - \\
\hline 26057 & $1.34 \times 10^{-5}$ & $3.63 \times 10^{-5}$ & - & $4.44 \times 10^{-5}$ & $4.47 \times 10^{-5}$ & - & $7.98 \times 10^{-5}$ & $4.10 \times 10^{-5}$ & - \\
\hline 26058 & $5.91 \times 10^{-8}$ & $5.87 \times 10^{-8}$ & - & $6.43 \times 10^{-7}$ & $6.71 \times 10^{-7}$ & - & $7.86 \times 10^{-7}$ & $7.83 \times 10^{-7}$ & - \\
\hline 28058 & $5.94 \times 10^{-8}$ & $5.93 \times 10^{-8}$ & - & $5.59 \times 10^{-7}$ & $6.66 \times 10^{-7}$ & - & $5.43 \times 10^{-7}$ & $7.14 \times 10^{-7}$ & - \\
\hline 28060 & $5.94 \times 10^{-8}$ & $5.93 \times 10^{-8}$ & - & $5.37 \times 10^{-7}$ & $8.36 \times 10^{-7}$ & - & $4.09 \times 10^{-7}$ & $8.78 \times 10^{-7}$ & - \\
\hline 28061 & $5.91 \times 10^{-8}$ & $5.93 \times 10^{-8}$ & - & $2.47 \times 10^{-7}$ & $4.18 \times 10^{-7}$ & - & $2.68 \times 10^{-7}$ & $5.23 \times 10^{-7}$ & - \\
\hline 28062 & $6.45 \times 10^{-8}$ & $2.53 \times 10^{-7}$ & - & $1.66 \times 10^{-7}$ & $6.66 \times 10^{-7}$ & - & $2.74 \times 10^{-7}$ & $8.01 \times 10^{-7}$ & - \\
\hline 28064 & $5.90 \times 10^{-8}$ & $5.99 \times 10^{-8}$ & - & $2.04 \times 10^{-7}$ & $3.19 \times 10^{-7}$ & - & $2.49 \times 10^{-7}$ & $3.26 \times 10^{-7}$ & - \\
\hline 29063 & $5.94 \times 10^{-8}$ & $1.27 \times 10^{-7}$ & - & $3.15 \times 10^{-7}$ & $6.27 \times 10^{-7}$ & - & $3.08 \times 10^{-7}$ & $6.64 \times 10^{-7}$ & - \\
\hline 29065 & $9.03 \times 10^{-8}$ & $5.99 \times 10^{-6}$ & - & $2.12 \times 10^{-7}$ & $5.99 \times 10^{-6}$ & - & $2.81 \times 10^{-7}$ & $5.99 \times 10^{-6}$ & - \\
\hline 40090 & $5.89 \times 10^{-8}$ & $5.87 \times 10^{-8}$ & - & $2.92 \times 10^{-7}$ & $5.95 \times 10^{-7}$ & - & $4.15 \times 10^{-7}$ & $7.25 \times 10^{-7}$ & - \\
\hline 40091 & $5.92 \times 10^{-8}$ & $5.90 \times 10^{-8}$ & - & $2.98 \times 10^{-7}$ & $6.23 \times 10^{-7}$ & - & $3.43 \times 10^{-7}$ & $7.19 \times 10^{-7}$ & - \\
\hline 40092 & $5.96 \times 10^{-8}$ & $5.91 \times 10^{-8}$ & - & $3.96 \times 10^{-7}$ & $7.48 \times 10^{-7}$ & - & $4.42 \times 10^{-7}$ & $8.26 \times 10^{-7}$ & - \\
\hline 40094 & $5.89 \times 10^{-8}$ & $5.93 \times 10^{-8}$ & - & $4.34 \times 10^{-7}$ & $7.34 \times 10^{-7}$ & - & $5.62 \times 10^{-7}$ & $7.48 \times 10^{-7}$ & - \\
\hline 40096 & $5.88 \times 10^{-8}$ & $5.79 \times 10^{-8}$ & - & $2.06 \times 10^{-7}$ & $5.46 \times 10^{-7}$ & - & $3.70 \times 10^{-7}$ & $6.69 \times 10^{-7}$ & - \\
\hline 42092 & $5.84 \times 10^{-8}$ & $5.93 \times 10^{-8}$ & - & $4.05 \times 10^{-7}$ & $6.62 \times 10^{-7}$ & - & $2.88 \times 10^{-7}$ & $7.85 \times 10^{-7}$ & - \\
\hline 42094 & $5.90 \times 10^{-8}$ & $5.95 \times 10^{-8}$ & - & $5.16 \times 10^{-7}$ & $7.26 \times 10^{-7}$ & - & $4.30 \times 10^{-7}$ & $8.61 \times 10^{-7}$ & - \\
\hline 42096 & $5.93 \times 10^{-8}$ & $5.89 \times 10^{-8}$ & - & $5.28 \times 10^{-7}$ & $7.99 \times 10^{-7}$ & - & $5.90 \times 10^{-7}$ & $8.93 \times 10^{-7}$ & - \\
\hline 42097 & $5.93 \times 10^{-8}$ & $5.94 \times 10^{-8}$ & - & $3.36 \times 10^{-7}$ & $4.86 \times 10^{-7}$ & - & $4.21 \times 10^{-7}$ & $6.00 \times 10^{-7}$ & - \\
\hline 42098 & $5.93 \times 10^{-8}$ & $8.80 \times 10^{-8}$ & - & $5.07 \times 10^{-7}$ & $8.01 \times 10^{-7}$ & - & $6.83 \times 10^{-7}$ & $9.17 \times 10^{-7}$ & - \\
\hline 42100 & $4.95 \times 10^{-6}$ & $2.86 \times 10^{-6}$ & - & $1.91 \times 10^{-6}$ & $5.44 \times 10^{-6}$ & - & $1.21 \times 10^{-6}$ & $8.33 \times 10^{-6}$ & - \\
\hline 47107 & $5.91 \times 10^{-8}$ & $5.94 \times 10^{-8}$ & - & $4.58 \times 10^{-7}$ & $6.39 \times 10^{-7}$ & - & $4.70 \times 10^{-7}$ & $6.96 \times 10^{-7}$ & - \\
\hline 47109 & $2.73 \times 10^{-5}$ & $1.76 \times 10^{-4}$ & - & $2.00 \times 10^{-5}$ & $1.69 \times 10^{-4}$ & - & $1.63 \times 10^{-5}$ & $1.28 \times 10^{-4}$ & - \\
\hline 48106 & $5.94 \times 10^{-8}$ & $5.94 \times 10^{-8}$ & - & $5.22 \times 10^{-7}$ & $6.25 \times 10^{-7}$ & - & $6.11 \times 10^{-7}$ & $7.22 \times 10^{-7}$ & - \\
\hline 48108 & $5.92 \times 10^{-8}$ & $5.93 \times 10^{-8}$ & - & $5.77 \times 10^{-7}$ & $6.65 \times 10^{-7}$ & - & $7.36 \times 10^{-7}$ & $7.70 \times 10^{-7}$ & - \\
\hline 48110 & $5.89 \times 10^{-8}$ & $5.92 \times 10^{-8}$ & - & $6.36 \times 10^{-7}$ & $8.01 \times 10^{-7}$ & - & $6.78 \times 10^{-7}$ & $8.93 \times 10^{-7}$ & - \\
\hline 48111 & $5.89 \times 10^{-8}$ & $5.93 \times 10^{-8}$ & - & $4.65 \times 10^{-7}$ & $5.59 \times 10^{-7}$ & - & $4.86 \times 10^{-7}$ & $6.56 \times 10^{-7}$ & - \\
\hline
\end{tabular}




\begin{tabular}{|c|c|c|c|c|c|c|c|c|c|}
\hline 48112 & $5.94 \times 10^{-8}$ & $5.94 \times 10^{-8}$ & - & $5.95 \times 10^{-7}$ & $7.87 \times 10^{-7}$ & - & $6.80 \times 10^{-7}$ & $8.77 \times 10^{-7}$ & - \\
\hline 48113 & $8.20 \times 10^{-6}$ & $1.37 \times 10^{-4}$ & - & $5.94 \times 10^{-6}$ & $1.34 \times 10^{-4}$ & - & $5.11 \times 10^{-6}$ & $1.18 \times 10^{-4}$ & - \\
\hline 48114 & $5.92 \times 10^{-8}$ & $5.92 \times 10^{-8}$ & - & $6.16 \times 10^{-7}$ & $8.57 \times 10^{-7}$ & - & $7.13 \times 10^{-7}$ & $9.57 \times 10^{-7}$ & - \\
\hline 48116 & $5.81 \times 10^{-8}$ & $5.92 \times 10^{-8}$ & - & $6.54 \times 10^{-7}$ & $8.68 \times 10^{-7}$ & - & $6.99 \times 10^{-7}$ & $9.88 \times 10^{-7}$ & - \\
\hline 49113 & $5.90 \times 10^{-8}$ & $5.95 \times 10^{-8}$ & - & $4.32 \times 10^{-7}$ & $5.22 \times 10^{-7}$ & - & $4.75 \times 10^{-7}$ & $6.52 \times 10^{-7}$ & - \\
\hline 49115 & $6.45 \times 10^{-8}$ & $3.65 \times 10^{-7}$ & - & $4.05 \times 10^{-7}$ & $5.45 \times 10^{-7}$ & - & $4.13 \times 10^{-7}$ & $6.00 \times 10^{-7}$ & - \\
\hline 50112 & $5.87 \times 10^{-8}$ & $5.79 \times 10^{-8}$ & - & $4.62 \times 10^{-7}$ & $4.63 \times 10^{-7}$ & - & $5.99 \times 10^{-7}$ & $5.83 \times 10^{-7}$ & - \\
\hline 50114 & $5.90 \times 10^{-8}$ & $5.76 \times 10^{-8}$ & - & $5.63 \times 10^{-7}$ & $6.03 \times 10^{-7}$ & - & $6.03 \times 10^{-7}$ & $7.30 \times 10^{-7}$ & - \\
\hline 50115 & $5.75 \times 10^{-8}$ & $5.67 \times 10^{-8}$ & - & $4.30 \times 10^{-7}$ & $3.76 \times 10^{-7}$ & - & $4.61 \times 10^{-7}$ & $5.16 \times 10^{-7}$ & - \\
\hline 50116 & $5.94 \times 10^{-8}$ & $5.95 \times 10^{-8}$ & - & $6.39 \times 10^{-7}$ & $9.36 \times 10^{-7}$ & - & $6.73 \times 10^{-7}$ & $1.03 \times 10^{-6}$ & - \\
\hline 50117 & $5.91 \times 10^{-8}$ & $5.93 \times 10^{-8}$ & - & $5.27 \times 10^{-7}$ & $5.54 \times 10^{-7}$ & - & $4.96 \times 10^{-7}$ & $6.63 \times 10^{-7}$ & - \\
\hline 50118 & $5.73 \times 10^{-8}$ & $5.82 \times 10^{-8}$ & - & $6.30 \times 10^{-7}$ & $6.76 \times 10^{-7}$ & - & $6.83 \times 10^{-7}$ & $8.35 \times 10^{-7}$ & - \\
\hline 50119 & $5.86 \times 10^{-8}$ & $5.95 \times 10^{-8}$ & - & $4.20 \times 10^{-7}$ & $5.76 \times 10^{-7}$ & - & $5.11 \times 10^{-7}$ & $6.79 \times 10^{-7}$ & - \\
\hline 50120 & $5.90 \times 10^{-8}$ & $1.84 \times 10^{-7}$ & - & $6.39 \times 10^{-7}$ & $9.79 \times 10^{-7}$ & - & $7.07 \times 10^{-7}$ & $1.09 \times 10^{-6}$ & - \\
\hline 50122 & $6.00 \times 10^{-8}$ & $6.89 \times 10^{-6}$ & - & $5.20 \times 10^{-7}$ & $6.89 \times 10^{-6}$ & - & $4.91 \times 10^{-7}$ & $6.89 \times 10^{-6}$ & - \\
\hline 50124 & $7.79 \times 10^{-6}$ & $8.85 \times 10^{-5}$ & - & $2.89 \times 10^{-6}$ & $8.85 \times 10^{-5}$ & - & $1.70 \times 10^{-6}$ & $8.85 \times 10^{-5}$ & - \\
\hline 92234 & $5.91 \times 10^{-8}$ & $5.93 \times 10^{-8}$ & $8.14 \times 10^{-8}$ & $6.52 \times 10^{-7}$ & $7.53 \times 10^{-7}$ & $8.07 \times 10^{-7}$ & $6.99 \times 10^{-7}$ & $8.44 \times 10^{-7}$ & $9.07 \times 10^{-7}$ \\
\hline 92235 & $1.69 \times 10^{-7}$ & $9.87 \times 10^{-7}$ & $6.04 \times 10^{-6}$ & $2.72 \times 10^{-7}$ & $4.91 \times 10^{-7}$ & $7.91 \times 10^{-7}$ & $2.81 \times 10^{-7}$ & $3.64 \times 10^{-7}$ & $7.53 \times 10^{-7}$ \\
\hline 92238 & $5.94 \times 10^{-8}$ & $5.96 \times 10^{-8}$ & $6.59 \times 10^{-7}$ & $6.64 \times 10^{-7}$ & $9.47 \times 10^{-7}$ & $1.17 \times 10^{-6}$ & $6.85 \times 10^{-7}$ & $9.56 \times 10^{-7}$ & $1.28 \times 10^{-6}$ \\
\hline
\end{tabular}




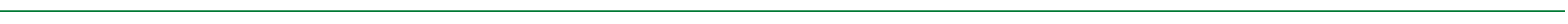

